\title{
Mechanical Properties of Acellular Peripheral Nerve
}

\author{
Gregory H. Borschel, M.D.,* Kevin F. Kia,* William M. Kuzon, Jr., M.D., Ph.D.,* \\ and Robert G. Dennis, Ph.D. $\dagger^{1}$ \\ *University of Michigan Section of Plastic Surgery, Ann Arbor, Michigan; and $\dagger$ Department of Mechanical Engineering, \\ University of Michigan, 2350 Hayward, Room 3116, Ann Arbor, Michigan
}

Submitted for publication September 25, 2002

Background. Acellular nerve has been used in experimental models as a peripheral nerve substitute. Our objective was to determine the difference in tensile strength between fresh and chemically treated acellularized peripheral nerve.

Materials and methods. F344 rat sciatic nerves were either fresh or acellularized and tested either whole (Part A) or transected and repaired (Part B). For all constructs, the mean ultimate stress, mean ultimate strain, Young's modulus, and total mechanical work to fracture were calculated.

Results. The average ultimate strains for Groups A-1 and A-2 were $0.480 \pm 0.117$ and $0.810 \pm 0.114$, respectively. The Young's moduli in Groups A-1 and A-2 were $576 \pm 160$ and $580 \pm 150 \mathrm{kPa}$, respectively. In Groups A-1 and A-2, the normalized work to failure was $0.35 \pm$ 0.14 and $1.11 \pm 0.38 \mathrm{~N}$. The specimens in Group B-1 withstood an average ultimate stress of $780 \pm 280 \mathrm{kPa}$. The specimens in Group B-2 withstood an average ultimate stress of $405 \pm 20 \mathrm{kPa}$. The average ultimate strains for Groups B-1 and B-2 were $0.319 \pm 0.087$ and $0.266 \pm 0.019$, respectively. The Young's moduli in Groups B-1 and B-2 were 4,030 \pm 1360 and 2,290 \pm 280 $\mathrm{kPa}$, respectively. The normalized work to failure in Groups B-1 and B-2 was calculated as $0.22 \pm 0.04$ and $0.11 \pm 0.02 \mathrm{~N}$.

Conclusions. Although adequately robust for reconstructive procedures, the acellular peripheral nerve had decreased tensile strength compared with fresh nerve either when tested whole or when transected and repaired. ๑ 2003 Elsevier Inc. All rights reserved.

Key Words: acellular nerve; tissue engineering; peripheral nerve; extracellular matrix; nerve regenera-

\footnotetext{
${ }^{1}$ To whom correspondence and reprint requests should be addressed at the Department of Mechanical Engineering, the University of Michigan, 2350 Hayward, Room 3116, Ann Arbor, MI 481092125. Fax: +1-734-936-2116. E-mail: bobden@umich.edu.
}

tion; nerve graft; nerve repair; biomechanics; tensile strength; mechanical properties.

\section{INTRODUCTION}

Autologous peripheral nerve grafting remains the "gold standard" in cases of nerve loss secondary to trauma or surgical excision. A readily available alternative to autologous nerve grafting would be of significant benefit to reconstructive surgeons and their patients. Substantially larger segmental defects could be reconstructed, and the morbidity of harvesting would be eliminated. Several types of materials have been proposed as peripheral nerve substitutes [1-15], including autologous materials such as vein and muscle strips, synthetic materials such as polylactic acid and polyglycolic acid, non-autologous biological materials such as acellular peripheral nerve allograft, and conduits made of collagen, laminin, fibronectin, and alginate.

In the "classical" tissue-engineering paradigm, a microporous matrix is seeded with cells to form a new tissue. One strategy used in our laboratory consists of using peripheral nerve extracellular matrix as a natural scaffold to support endogenous cellular growth. Our laboratory has previously demonstrated that axonal growth occurs in acellular peripheral nerve allograft in vivo [16]. In addition to supporting axonal growth, it is important for a peripheral nerve substitute to have sufficient tensile strength and mechanical toughness to withstand in vivo mechanical forces. Investigation of tensile properties is a requisite step in the further development of peripheral nerve substitutes. The purpose of this study was to determine the tensile properties of acellular peripheral nerve compared with fresh nerve by measuring tensile load at failure, total mechanical work to tensile failure, and mechanical strain at failure at a fixed rate of elongation. 


\section{TABLE 1}

\section{Chemical Acellularization Protocol}

\begin{tabular}{lc}
\hline \multicolumn{1}{c}{ Reagent } & Time, days \\
\hline 80\% glycerol/0.9\% NaCl/0.05\% $\mathrm{NaN}_{3} / 25 \mathrm{mM}$ EDTA & 3 \\
Na deoxycholate $4.2 \% / 0.05 \% \mathrm{NaN}_{3}$ & 3 \\
$80 \%$ glycerol/0.9\% NaCl/0.05\% $\mathrm{NaN}_{3} / 25 \mathrm{mM}$ EDTA & 2 \\
$1 \% \mathrm{SDS} / 0.05 \% \mathrm{NaN}_{3}$ & 2 \\
$3 \%$ Triton X-100/0.05\% $\mathrm{NaN}_{3}$ & 2 \\
$1 \% \mathrm{SDS} / 0.05 \% \mathrm{NaN}_{3}$ & 2 \\
$0.05 \% \mathrm{NaN}_{3}$ & 2 \\
\hline
\end{tabular}

\section{METHODS}

Approval for animal use was granted by the University Committee for the Use and Care of Animals at the University of Michigan. Ketamine $100 \mathrm{mg} / \mathrm{kg}$ and xylazine $10 \mathrm{mg} / \mathrm{kg}$ were used for general anesthesia. The sciatic nerves of adult F344 rats (Charles River Labs, Wilmington, MA) were harvested. Care was taken during the dissection to minimize dessication and stretching of the nerves.

F344 rat sciatic nerves were either fresh or acellularized, and were tested either whole or transected and repaired. In the first part of the experiment (Part A), acellular nerves (Group A-1) and fresh nerves (Group A-2) were tested whole. In the second part of the experiment (Part B), fresh nerves were transected and coapted (Group B-1) with six 10-0 epineurial nylon sutures. In Group B-2, both nerve types (acellular and fresh) were coapted to simulate the use of the acellular nerve as a graft. Four specimens were tested per group, for a total of 16 specimens.

\section{Acellularization}

Acellularization was carried out by using a previously developed protocol [15]. Fresh nerves were pinned onto 100-mm Petri dishes coated with silicone elastomer (Sylgard ${ }^{\circledR}$, Dow Corning, Midland, MI). The nerves were then treated with a series of detergent baths (Table 1) consisting of glycerol, sodium deoxycholate, sodium dodecyl sulfate, and Triton X-100 (all reagents from Fisher Scientific, Pittsburgh, PA). All acellularization steps were performed at room temperature.

\section{Tensile Testing}

After the acellularization process was complete, the nerves were loaded onto a tensile testing device that was designed and built in our laboratory. The device consisted of a linear stepper motor with a microstepping driver (Hurst Manufacturing, Princeton, IN) and an embedded microcontroller. The strain rate was set to $1 \% / \mathrm{s}$. Nerve specimens were attached to the tensile tester via stainless steel hooks by using 4-0 silk sutures and ethyl cyanoacrylate adhesive (Super Glue ${ }^{\mathrm{TM}}$, Elmer's Products, Columbus, OH). We used the system of hooks and sutures with glue to ensure that no slippage occurred during testing. We monitored the testing under a dissecting microscope to ensure that slippage did not occur. The nerves burst at a location away from the points of connection. In some cases, the constructs failed at the point of connection, and these data were not included in the study.

Samples were kept moist during testing by applying a drop of normal saline solution to the nerve constructs. Each specimen was stretched at a constant rate of $0.11 \mathrm{~mm} / \mathrm{s}$. The average specimen length was $13.72 \pm 0.44 \mathrm{~mm}$. Force and displacement were sampled twice per second. Each sample was stretched to complete tensile failure.

\section{Data Acquisition and Analysis}

For all constructs, the burst strength (force at failure), mean ultimate stress, mean ultimate strain, Young's modulus, and total work to produce a failure were determined from the initial length and thickness of the specimens and the force tracings measured during testing. An example of such a stress-strain curve is shown in Fig. 1. Ultimate stress refers to the amount of force per unit of initial cross-sectional area at tensile failure. Ultimate strain refers to the amount of elongation divided by the initial specimen length achieved at the point of tensile failure. Young's modulus (a measure of material stiffness) was calculated from the slope of the ascending portion of the stress-strain curve after normalizing for the specimen geometry. The work to produce a tensile failure was calculated by numerical integration of the area under the load-deformation curve. The normalized work to failure was calculated by dividing the work to failure by the length of each specimen. Calculating the normalized work to failure provided a means of directly comparing groups of specimens by correcting for minor variations in initial length.

The Wilcoxon rank sum test (SigmaStat v. 2.03, SPSS, Chicago, IL) was used to determine if there were differences between groups in ultimate stress and strain, Young's modulus, and work to failure. Group A-1 was compared to Group A-2, and Group B-1 was compared to Group B-2. Alpha was set a priori at a level of 0.05. For each result, the mean \pm the standard deviation is reported.

\section{Electron Microscopy}

Normal and acellular nerves were pinned at slack length and fixed for $4 \mathrm{~h}$ at $4^{\circ} \mathrm{C}$ in Karnovsky's solution $(0.1 \mathrm{M}$ sodium cacodylate buffer with $3 \%$ formaldehyde and $3 \%$ glutaraldehyde, $\mathrm{pH} 7.4$ ). Constructs were rinsed 3 times ( $30 \mathrm{~min}, 30 \mathrm{~min}$, and $4 \mathrm{~h}$ ) with cacodylate (pH 7.4) with 7.5\% sucrose. Constructs were postfixed in $1 \%$ osmium tetroxide for $2 \mathrm{~h}$ at room temperature, dehydrated in graded concentrations of ethanol and propylene oxide, and embedded in EPON (Eponate 12 resin, Ted Pella, Redding, CA) for electron microscopy. Transmission electron micrographs were obtained at magnifications of $4290-54,600 \times$ on a Philips CM-100 electron microscope at $60 \mathrm{kV}$.

\section{RESULTS}

There were no significant differences in the average lengths and widths of the specimens as tested. Speci-

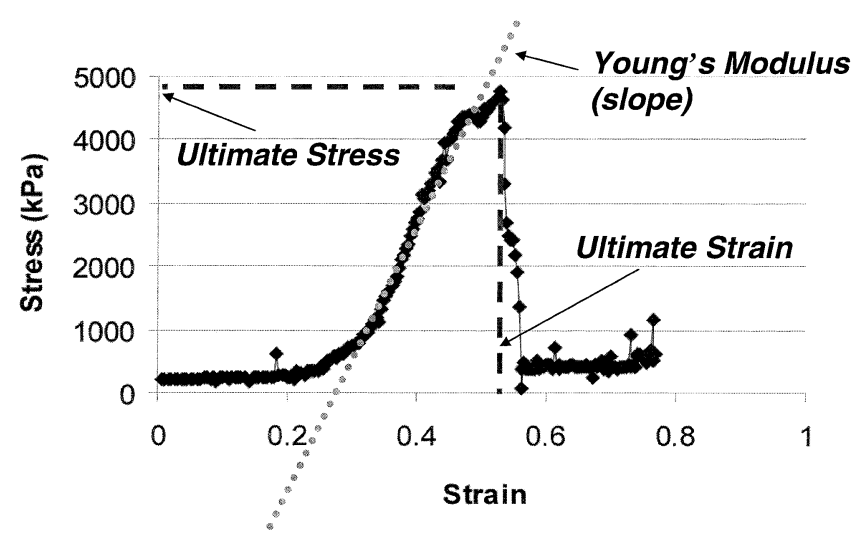

FIG. 1. Stress-strain curve for a fresh, whole specimen demonstrating ultimate stress and strain, and Young's modulus of elasticity (slope of ascending portion of curve). The work to produce a failure is calculated from the area under the load-deformation curve (not shown). 
TABLE 2

Results for Groups A-1 and A-2

\begin{tabular}{|c|c|c|c|c|c|c|}
\hline Group & $\begin{array}{c}\text { Burst strength } \\
\text { (force at failure), } \mathrm{N}\end{array}$ & $\begin{array}{c}\text { Ultimate } \\
\text { stress, } \mathrm{kPa}\end{array}$ & $\begin{array}{l}\text { Ultimate } \\
\text { strain }\end{array}$ & Young's modulus, $\mathrm{kPa}$ & $\begin{array}{c}\text { Work to } \\
\text { failure, Nmm }\end{array}$ & $\begin{array}{l}\text { Normalized work } \\
\text { to failure, } \mathrm{N}\end{array}$ \\
\hline A-1 & $1.10 \pm 0.23$ & $1400 \pm 290$ & $0.480 \pm 0.117^{*}$ & $576 \pm 160$ & $3.93 \pm 1.71^{*}$ & $0.35 \pm 0.14^{*}$ \\
\hline A-2 & $2.14 \pm 0.76$ & $2720 \pm 970$ & $0.810 \pm 0.114$ & $580 \pm 150$ & $12.73 \pm 5.10$ & $1.11 \pm 0.38$ \\
\hline
\end{tabular}

Note. All data are displayed with standard deviations. The asterisk $(*)$ indicates a statistically significant difference between groups A-1 and A-2 $(P<0.05$ by the Wilcoxon rank sum test $)$.

mens failed between the restraining hooks in Groups A-1 and A-2. All specimens failed at the site of coaptation in Groups B-1 and B-2. No loosening of sutures or slipping of specimens was observed during testing.

\section{Part A: Whole Specimens (Table 2)}

In Part A, specimens from Groups A-1 and A-2 were tested whole. The specimens in Group A-1 withstood an average ultimate stress of $1400 \pm 290 \mathrm{kPa}$. The specimens in Group A-2 were able to withstand an average ultimate stress of $2720 \pm 970 \mathrm{kPa}$. The difference in ultimate stresses approached statistical significance by the Wilcoxon rank sum test $(P=0.057)$. The average ultimate strains for Groups A-1 and A-2 were $0.480 \pm 0.117$ and $0.810 \pm 0.114$, respectively; this difference was statistically significant $(P=0.007)$. The Young's moduli in Groups A-1 and A-2 were $576 \pm 160$ and $580 \pm 150 \mathrm{kPa}$, respectively. No statistically significant difference in Young's modulus was detected between Groups A-1 and A-2. Mechanical work to failure was normalized by dividing by the initial specimen length. In Groups A-1 and A-2, the normalized work to failure was $0.35 \pm 0.14 \mathrm{~N}$ and $1.11 \pm 0.38 \mathrm{~N}(P=0.029$ between Groups A-1 and A-2). Work to failure is expressed as Newton-meters; dividing the work to failure by the specimen length results in the normalized work to failure in Newtons. The burst strength, or force attained upon failure, was $1.10 \pm 0.23$ and $2.14 \pm 0.76$ $\mathrm{N}$ for Groups A-1 and A-2, respectively.

\section{Part B: Specimens with Coaptations (Table 3)}

In Part B, Groups B-1 and B-2 were compared. The specimens in Group B-1 withstood an average ultimate stress of $780 \pm 280 \mathrm{kPa}$. The specimens in Group B-2 withstood an average ultimate stress of $405 \pm 20 \mathrm{kPa}$. The difference in ultimate stresses was statistically significant at a level of $P<0.05$. The average ultimate strains for Groups B-1 and B-2 were $0.319 \pm 0.087$ and $0.266 \pm 0.019$, respectively; however, this difference was not statistically significant $(P=0.34)$. The Young's moduli in Groups B-1 and B-2 were $4030 \pm 1360$ and $2290 \pm 280 \mathrm{kPa}$, respectively (no significant difference; $P=0.11$ ). The normalized work to failure in Groups B-1 and B-2 was calculated as $0.22 \pm 0.04$ and $0.11 \pm$ $0.02 \mathrm{~N}$, respectively. The difference in normalized work to failure was statistically significant $(P=0.029)$. The burst strength was $0.61 \pm 0.22$ and $0.41 \pm 0.02 \mathrm{~N}$ for Groups B-1 and B-2, respectively $(P<0.05)$.

\section{Electron Microscopy}

Electron microscopy of the acellularized nerves demonstrated uniform lack of cells throughout the treated nerves (Figs. 2 and 3). Normal cellular structures found in peripheral nerve, including axons, Schwann cell bodies, and myelin, were absent in the acellular nerve material. Vacant endoneurial conduits were seen within a lattice of extracellular matrix. The resulting endoneurial conduits measured 2 to $6 \mu$ in diameter.

\section{DISCUSSION}

The tensile strength of peripheral nerve was overall decreased by the acellularization process. The ultimate strain was decreased relative to fresh nerve. The mean ultimate stress of the acellular nerve (burst strength normalized for cross-sectional area) was less than that

TABLE 3

Results for Groups B-1 and B-2

\begin{tabular}{|c|c|c|c|c|c|c|}
\hline Group & $\begin{array}{c}\text { Burst strength } \\
\text { (force at failure), } \mathrm{N}\end{array}$ & $\begin{array}{l}\text { Ultimate } \\
\text { stress, } \mathrm{kPa}\end{array}$ & $\begin{array}{l}\text { Ultimate } \\
\text { strain }\end{array}$ & Young's modulus, $\mathrm{kPa}$ & $\begin{array}{c}\text { Work to } \\
\text { failure, Nmm }\end{array}$ & $\begin{array}{l}\text { Normalized work } \\
\text { to failure, } \mathrm{N}\end{array}$ \\
\hline B-1 & $0.61 \pm 0.22^{*}$ & $780 \pm 280 *$ & $0.319 \pm 0.087$ & $4030 \pm 1360$ & $3.69 \pm 1.81^{*}$ & $0.22 \pm 0.04^{*}$ \\
\hline B-2 & $0.41 \pm 0.02$ & $405 \pm \quad 20$ & $0.266 \pm 0.019$ & $2290 \pm 280$ & $1.25 \pm 0.19$ & $0.11 \pm 0.02$ \\
\hline
\end{tabular}

Note. All data are displayed with standard deviations. The asterisk $(*)$ indicates a statistically significant difference between groups B-1 and B-2 $(P<0.05$ by the Wilcoxon rank sum test). 


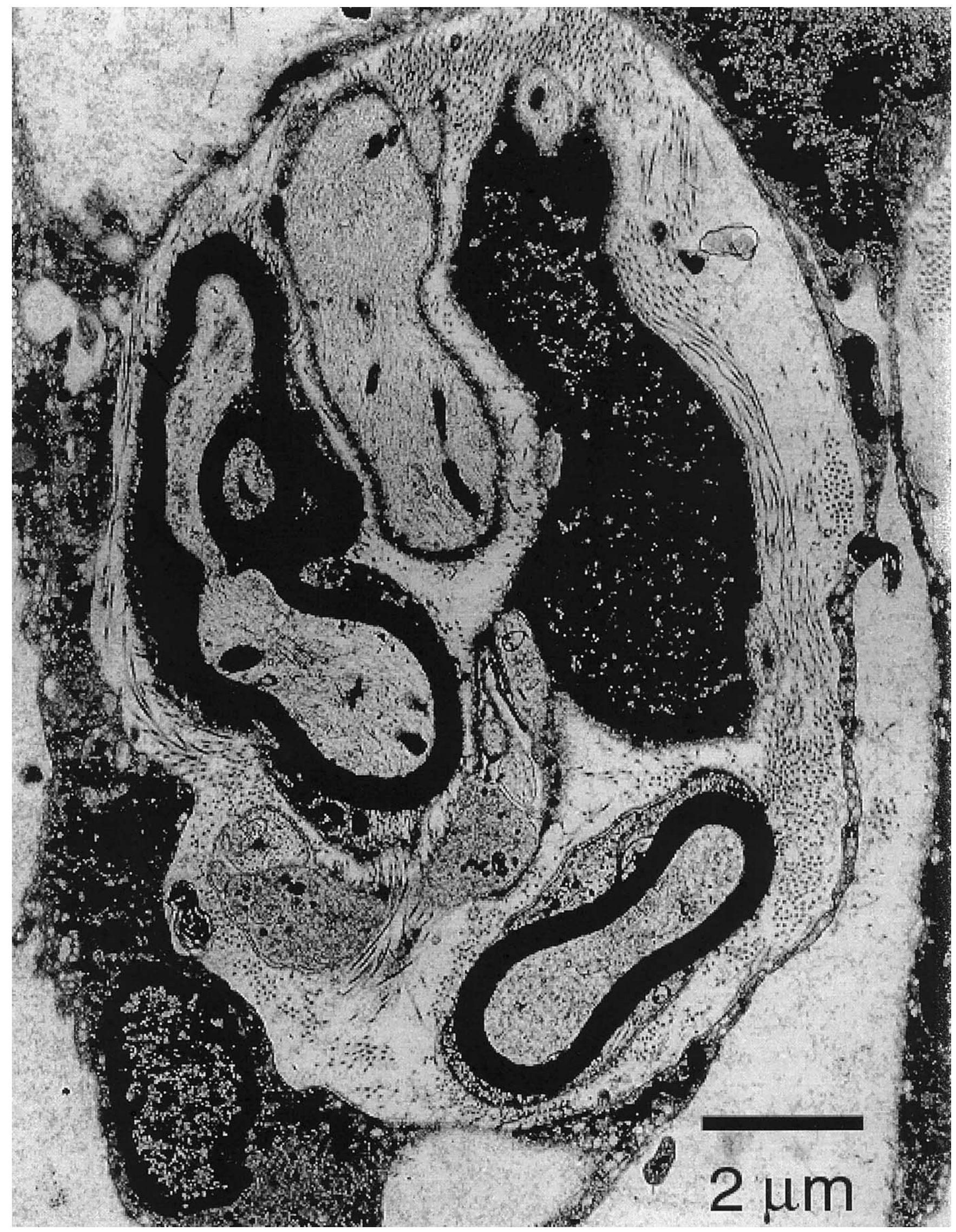

FIG. 2. Transmission electron micrograph of normal rat peripheral nerve, with darkly stained myelinated axons and Schwann cells. 


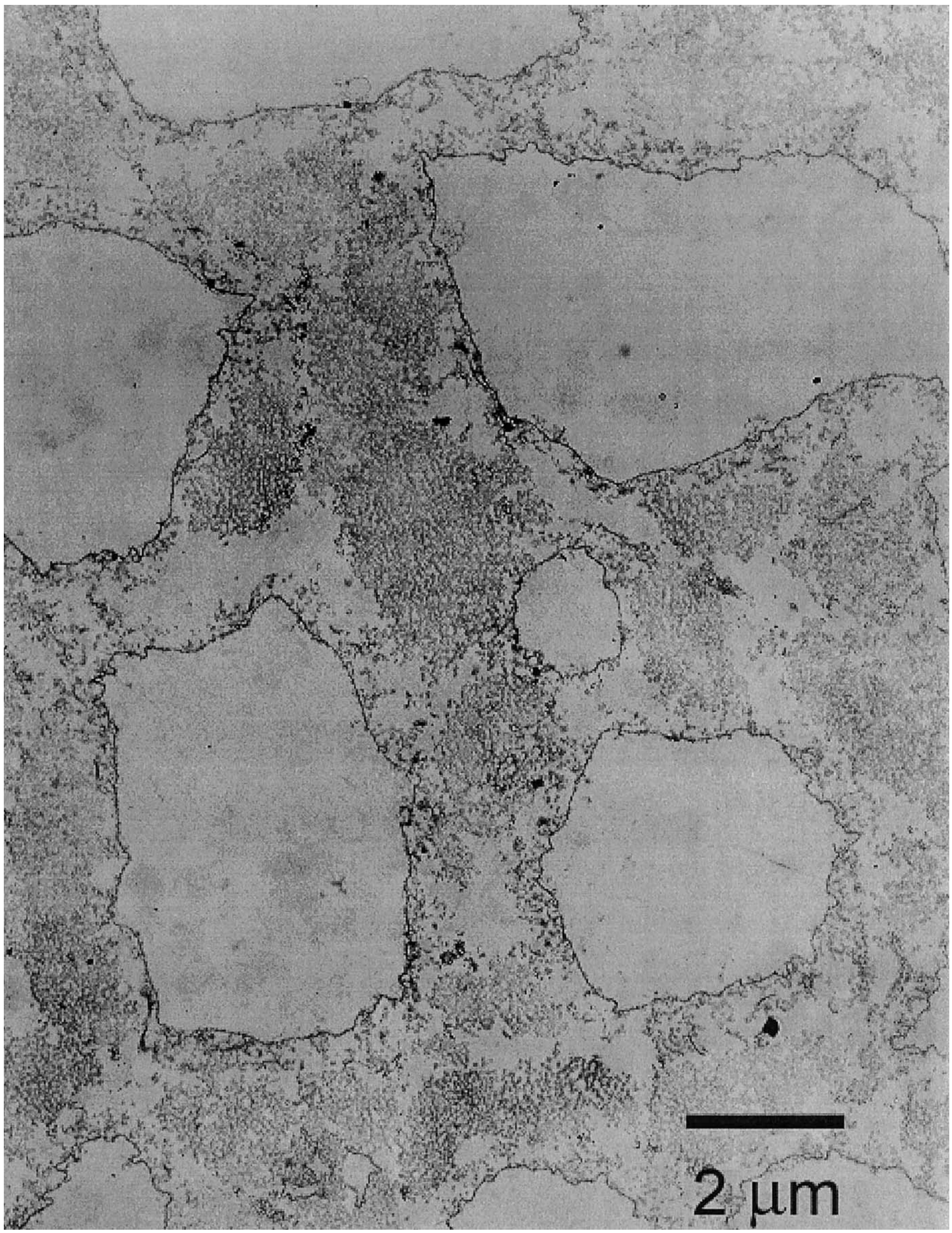

FIG. 3. Electron microscopy of the acellularized nerves demonstrated uniform lack of cells throughout the treated nerves. Compared with normal nerve, features such as myelin, axons, Schwann cells, vessels, and other cellular elements were absent. Vacant endoneurial conduits, measuring 2 to $6 \mu$ in diameter, remained patent. 
of fresh nerve, but this difference was not significantly significant by the Wilcoxon rank sum test. No significant difference was noted in the Young's modulus, indicating that the stiffness of the materials was equivalent.

A statistically significant difference was noted in the normalized work to failure for Groups A-1 and A-2. The normalized work to failure is an excellent metric of tensile strength. Taken as a whole, the findings from Part A indicate that the tensile strength of acellular peripheral nerve was decreased relative to fresh nerve.

In Part B, ultimate stress and normalized work to failure were decreased relative to fresh nerve. The results from Part B are explained partly by the fact that the constructs in Groups B-1 and B-2 were composed partly of nylon suture. Because nylon is stiff, i.e., has a relatively high Young's modulus, the coapted constructs would be predicted to have increased stiffness. The stiffness of a composite material, such as the nerve-suture construct, is dependent on all components of the composite, as well as their disposition within the composite, and the mechanical coupling between these materials at their interfaces. For simple springs in series, the total stiffness, $K$, is equal to the inverse of the sum of the inverse stiffnesses; thus, the addition of any series-compliant element necessarily reduces the total structural stiffness. In comparison, elastic elements added in parallel add linearly to stiffness, so the addition of any parallel elastic elements always increases the total stiffness of a structure. In the case of our constructs, the sutures lie parallel to the soft-tissue structures along some of their length, so the addition of sutures will tend to increase the total stiffness of the structure. Likewise, differences in the ultimate stress and strain between Groups B-1 and B-2 may have been masked by the contribution of nylon suture in the constructs. Nevertheless, the normalized work to failure in the acellular group was half that of the fresh group. The findings from Part B indicate overall that the tensile strength of acellular peripheral nerve is decreased relative to fresh nerve in the presence of a coaptation.

This work has applied standard materials testing methods to evaluate an engineered peripheral nerve replacement. Four distinct tensile characteristics were evaluated (ultimate stress and strain, Young's modulus, and work to failure). It is necessary to evaluate such characteristics of any material designed for implantation in patients.

Some observations can be made in comparison with data from rabbit tibial nerve. Under similar experimental conditions, it was found that fresh rabbit tibial nerve had an ultimate stress of $11700 \pm 700 \mathrm{kPa}$ and an ultimate strain of $0.385 \pm 0.020$ [17]. Another experiment documented an ultimate strain of 0.557 [18]. The ultimate stress of the rabbit tibial nerve was much higher than the rat sciatic nerve. This finding may be caused by the fact that as the number of fascicles in a nerve increases, the tensile strength increases more than would be expected $[19,20]$. The ultimate strain of the rat sciatic nerve was greater than the ultimate strain reported for rabbit tibial nerve; it is possible that the rat nerve had less total extracellular matrix (ECM) per unit volume, resulting in a more pliable composite material over the tested strains.

The strongest connective tissue layers in peripheral nerves are the perineurium and, to a lesser extent, the epineurium [21]. Alterations in the epineurium and perineurium ECM composition are likely to have significant effects on the tensile strength of acellular nerve constructs. It is likely that chemical acellularization decreases the amounts of certain ECM molecules. The acellularization process may have partially removed one or more components of the ECM in a way that reduced the amount of energy able to be absorbed before tensile failure. Additional experiments are under way to quantify the degree of ECM composition change caused by the acellularization process. Additionally, other mechanical properties of peripheral nerves are important and merit investigation, such as compression strength [21].

With respect to mechanical properties, acellular nerve is appropriate for use in vivo. Nerve graft coaptations are tension free by design; however, in situ stress is always present in peripheral nerves, and this stress varies with joint position. For this reason, it is important to demonstrate in vitro that such a biomaterial has substantial tensile strength before embarking on clinical use. Properties such as suture-holding ability and, therefore, the ability to maintain a mechanically robust tissue interface with native nerve stump, are critical for axonal regeneration. Such properties depend largely on the tensile strength of the construct. Previous data showed that when acellular nerve was used in the rat hindlimb, the coaptations remained secure over several months [16]. The acellular nerve grafts are mechanically robust, hold sutures well during coaptation, and retain sutures well postoperatively.

\section{CONCLUSIONS}

Tensile testing of fresh and acellular nerve constructs demonstrated that, either when tested whole or with a coaptation, the tensile strength of the acellular nerve was decreased when compared to the fresh samples. Further research is currently directed toward the detailed characterization of the components of the acellular nerve, as well as in vivo testing of hydrogelmodified acellular nerve. 


\section{REFERENCES}

1. Toba, T., Nakamura, T., Lynn, A. K., Matsumoto, K., Fukuda, S., Yoshitani, M., Hori, Y., and Shimizu, Y. Evaluation of peripheral nerve regeneration across an $80-\mathrm{mm}$ gap using a polyglycolic acid (PGA)-collagen nerve conduit filled with lamininsoaked collagen sponge in dogs. Intl. J. Artif. Org. 25: 230, 2002.

2. Meek, M. F., and Coert, J. H. Clinical use of nerve conduits in peripheral-nerve repair: Review of the literature. J. Reconstruct. Microsurg. 18: 97, 2002.

3. Evans, G. R. D., Brandt, K., Katz, S., Chauvin, P., Otto, L., Bogle, M., Wang, B., Meszlenyi, R. K., Lu, L. C., Mikos, A. G., and Patrick, C. W. Bioactive poly(L-lactic acid) conduits seeded with Schwann cells for peripheral nerve regeneration. Biomaterials 23: 841, 2002.

4. Toba, T., Nakamura, T., Shimizu, Y., Matsumoto, K., Ohnishi, K., Fukuda, S., Yoshitani, M., Ueda, H., Hori, Y., and Endo, K. Regeneration of canine peroneal nerve with the use of a polyglycolic acid-collagen tube filled with laminin-soaked collagen sponge: a comparative study of collagen sponge and collagen fibers as filling materials for nerve conduits. J. Biomed. Mater. Res. 58: 622, 2001.

5. Watanabe, K., Tsukagoshi, T., Kuroda, M., and Hosaka, Y. Nerve conduit using fascia-wrapped fibrocollagenous tube. $J$. Reconstruct. Microsurg. 17: 363, 2001.

6. Sufan, W., Suzuki, Y., Tanihara, M., Ohnishi, K., Suzuki, K., Endo, K., and Nishimura, Y. Sciatic nerve regeneration through alginate with tubulation or nontubulation repair in cat. J. Neurotrauma 18: $329,2001$.

7. Valero-Cabre, A., Tsironis, K., Skouras, E., Perego, G., Navarro, X., and Neiss, W. F. Superior muscle reinnervation after autologous nerve graft or poly-L-lactide-epsilon-caprolactone (PLC) tube implantation in comparison to silicone tube repair. J. Neurosci. Res. 63: 214, 2001.

8. Kitahara, A. K., Nishimura, Y., Shimizu, Y., and Endo, K. Facial nerve repair accomplished by the interposition of a collagen nerve guide. J. Neurosurg. 93: 113, 2000.

9. Matsumoto, K., Ohnishi, K., Kiyotani, T., Sekine, T., Ueda, H., Nakamura, T., Endo, K., and Shimizu, Y. Peripheral nerve regeneration across an $80-\mathrm{mm}$ gap bridged by a polyglycolic acid (PGA)-collagen tube filled with laminin-coated collagen fibers: a histological and electrophysiological evaluation of regenerated nerves. Brain Res. 868: 315, 2000.

10. Fansa, H., Keilhoff, G., Forster, G., Seidel, B., Wolf, G., and Schneider, W. Acellular muscle with Schwann-cell implanta- tion: An alternative biologic nerve conduit. J. Reconstruct. Microsurg. 15: 531, 1999.

11. Evans, G. R. D., Brandt, K., Widmer, M. S., Lu, L., Meszlenyi, R. K., Gupta, P. K., Mikos, A. G., Hodges, J., Williams, J., Gurlek, A., Nabawi, A., Lohman, R., and Patrick, C. W. In vivo evaluation of poly(L-lactic acid) porous conduits for peripheral nerve regeneration. Biomaterials 20: 1109, 1999.

12. Whitworth, I. H., Brown, R. A., Dore, C., Green, C. J., and Terenghi, G. Orientated mats of fibronectin as a conduit material for use in peripheral-nerve repair. J. Hand Surg. (Br. Euro. Vol.) 20B: 429, 1995.

13. Chiu, D. T. W., and Strauch, B. A prospective clinical evaluation of autogenous vein grafts used as a nerve conduit for distal sensory nerve defects of $3 \mathrm{~cm}$ or less. Plastic Reconstruct. Surg. 86: $928,1990$.

14. Feinberg, J. H., Khouri, R. K., Spielholz, N., Harper, A., and Chiu, D. T. W. Nerve regeneration through an autologous venous nerve-conduit - the effects of conduit diameter and neurite promoting factors on motor action-potentials. Arch. Phys. Med. Rehabil. 69: 704, 1988.

15. Rovak, J. M., Boxer, L. K., Bishop, D. K., Chan, S. Y., Faulkner, J. A., Kuzon, W. M., Mungara, A. K., and Cederna, P. S. Th1 and Th2 responses to peripheral nerve transplantation: The role of chemical acellularization in eliminating allograft antigenicity. Plastic Reconstruct. Surg., in press.

16. Haase, S. C., Rovak, J. M., Dennis, R. G., Kuzon, W. M., and Cederna, P. S. Recovery of muscle contractile function following nerve gap repair with chemically acellularized peripheral nerve grafts. J. Reconstruct. Microsurg. 19: 241, 2003.

17. Rydevik, B. L., Kwan, M. K., Myers, R. R., Brown, R. A., Triggs, K. J., Woo, S. L. Y., and Garfin, S. R. An in vitro mechanical and histological study of acute stretching on rabbit tibial nerve. J. Orthop. Res. 8: 694, 1990.

18. Haftek, J. Stretch injury of peripheral nerve. Acute effects of stretching on rabbit nerve. J. Bone Joint Surg. Br. 52: 354, 1970.

19. Sunderland, S. The anatomy and physiology of nerve injury. Muscle Nerve 13: 771, 1990.

20. Sunderland, S. The connective tissues of peripheral nerves. Brain 88: 841, 1965

21. Rydevik, B. L., Lundborg, G., Olmarker, K., and Myers, R. R. Biomechanics of peripheral nerves and spinal nerve roots. in $\mathrm{M}$. Nordin, and V. H. Frankel (Eds.), Basic Biomechanics of the Musculoskeletal System. Baltimore: Lippincott Williams \& Wilkins, 2001, Pp. 126-146. 